\title{
The Influence of Thermal Expansion of a Composite Material on Embedded Polarimetric Sensors
}

\author{
Manjusha Ramakrishnan \\ Technological University Dublin \\ Ginu Rajan \\ Technological University Dublin, ginu.rajan@tudublin.ie \\ Yuliya Semenova \\ Technological University Dublin, yuliya.semenova@tudublin.ie
}

See next page for additional authors

Follow this and additional works at: https://arrow.tudublin.ie/engscheceart

Part of the Electrical and Computer Engineering Commons

\section{Recommended Citation}

Ramakrishnan, M. et al. (2011) The Influence of Thermal Expansion of a Composite Material on Embedded Polarimetric Sensors. Smart Materials and Structures, vol. 20(12):125002, pp.7. doi:10.1088/ 0964-1726/20/12/125002

This Article is brought to you for free and open access by the School of Electrical and Electronic Engineering at ARROW@TU Dublin. It has been accepted for inclusion in Articles by an authorized administrator of ARROW@TU Dublin. For more information, please contact arrow.admin@tudublin.ie, aisling.coyne@tudublin.ie, gerard.connolly@tudublin.ie. Funder: Enterprise Ireland under the international research grant MATERA ERA-NET

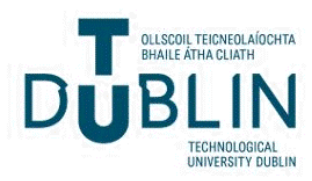


Authors

Manjusha Ramakrishnan, Ginu Rajan, Yuliya Semenova, Piotr Lesiak, Andrzej Domanski, Tomasz Wolinksi, Anna Boczkowska, and Gerald Farrell 


\title{
The influence of thermal expansion of a composite material on embedded polarimetric sensors
}

\author{
Manjusha Ramakrishnan ${ }^{1}$, Ginu Rajan $^{1}$, Yuliya Semenova ${ }^{1}$, Piotr Lesiak $^{2}$, \\ Andrzej Domanski ${ }^{2}$, Tomasz Wolinski ${ }^{2}$, Anna Boczkowska ${ }^{3}$ and Gerald \\ Farrell $^{1}$ \\ ${ }^{1}$ Photonics Research Centre, School of Electronic and Communications Engineering, Dublin \\ Institute of Technology, Kevin Street, Dublin 8, Ireland \\ ${ }^{2}$ Faculty of Physics, Warsaw University of Technology, Warszawa, Poland \\ ${ }^{3}$ Faculty of Materials Science and Engineering, Warsaw University of Technology, Warszawa, \\ Poland \\ *E-mail: ginu.rajan@dit.ie
}

\begin{abstract}
Some of the most critical issues of the influence of the thermal expansion of composite materials on embedded polarimetric sensors for measurements of strain and temperature are studied in this paper. A composite material sample with polarimetric fiber sensors embedded in two distinct layers of a multi-layer composite structure is fabricated and characterized. The polarimetric fiber sensors used in this study are based on panda type fiber and polarization maintaining photonic crystal fiber (PM-PCF). The temperature sensitivities of polarimetric fiber sensors with acrylate buffer coated and buffer stripped polarization maintaining optical fibers are measured in free space and compared to those for embedded in the composite material. It is found that a polarimetric fiber sensor with an acrylate coating embedded in the composite material shows the same response as the one in free space while the coating-stripped fiber polarimetric sensor shows significant temperature sensitivity when embedded in the composite material. This is due to the stress-induced change in birefringence created by the thermal expansion of the composite material, while in the case of a buffer coated fiber, the effect is considerably reduced as the thermal stress is largely eliminated by the buffer coating. The results obtained in this study demonstrated that thermal expansion of the composite material are the main source of error in strain and temperature measurement using embedded polarimetric fiber sensors and that more accurate strain and temperature measurements can be obtained with buffer coated polarimetric fiber sensors.
\end{abstract}

Keywords: Photonic crystal fiber sensors, polarimetric sensors, smart structures, composite materials

Submitted to: Smart Materials and Structures

\section{Introduction}

Composite material structures are widely used in the aerospace, marine, aviation and civil engineering industry, because of their superior properties [1,2]. Continuous fibre-reinforced composite structures $[3,4]$ have attractive properties such as low density, high strength and high stiffness. Additionally composite materials offer the potential to utilize embeded fiberoptic sensors (FOS) which make them suitable candidates for smart materials for various applications in aerospace components and civil engineering structures. One or more FOS embedded in a composite material can be used to monitor the condition of the material during the manufacturing process or alternatively to monitor the structural health of the composite part over its operational lifetime and thereby can enhance the reliability and safety of many advanced structures. Embedding FOS in composite materials is possible because their size and weight are comparable to those of the composite fibre reinforcement fabric, allowing 
them to act as a form of "nervous system" for a composite fiber matrix structure. Integrated FOSs offer many advantages over traditional nondestructive evaluation techniques and surface bonded sensors, such as more reliable data about matrix cracks, point damage and thermo-mechanical behavior.

The fiber optic sensor technology most widely used for structural health monitoring is fiber Bragg grating sensing [5]. However, the cross-sensitivity between temperature and strain is a serious disadvantage for many real-life applications. Polarimetric fiber sensing [6] technology is a promising candidate for sensing in composites as it offers temperature independence. Additionally given the availability of an extensive variety of polarization maintaining (PM) fibers and micro-structured highly-birefringent (HB) polarization maintaining fibers, such sensors possess a wide range of sensitivities and are suitable for sensing a variety of parameters, such as strain, temperature, displacement, humidity, etc independently. In comparison with previously reported methods for resolving the strain and temperature crosssensitivity issue with polarimetric sensors such as the fiber-optic differential heterodyne sensing scheme [7], and fiber-optic dual-technique sensor [8], the hybrid sensing approach described in [9] based on temperature insensitive PM-PCF offers the advantage of simplicity.

For composite structures most research reports have concentrated on the use of FBGs to measure temperature and strain during the curing process and during structural tests. A few authors have reported interferometric [10] and polarimetric sensors [11] embedded in composite materials. What has not been well examined in the previous research studies is the impact of the characteristic properties of the composite materials and also the effect of the fiber buffer coating on the characteristics of the embedded fiber sensors. In this paper a detailed investigation is carried out on the influence of thermo-mechanical properties of composite materials on the embedded polarimetric fiber sensors in order to explore the most suitable approach to embedding such sensors in composites. The objective of this research is to ensure consistent and accurate temperature or strain measurements for applications such as wind rotor blades and helicopter rotor blades which operate in varying atmospheric temperature conditions from -20 to $40{ }^{\circ} \mathrm{C}$. The polarimetric fiber sensors used are based on polarization maintaining photonic crystal fiber (PM-PCF) and panda fibers with and without an acrylate fiber buffer coating.

\section{Temperature sensitivity of polarimetric sensors}

In a polarimetric sensor [12], symmetric deformation or variation in the refractive index induced by temperature influences the propagation constant for every mode because of the changes in the fiber length and the refractive indices of the core and the cladding. This in turn leads to changes in the phase difference between the two orthogonal polarization states of the fundamental mode.

We can calculate the temperature sensitivity (S) of a $\mathrm{HB}$ fiber section with a length $\mathrm{L}$ as follows:

$$
S=\frac{2 \pi}{T_{T} \cdot L}\left[\mathrm{rad} /\left({ }^{0} \mathrm{C} \cdot \mathrm{m}\right)\right]
$$

where $T_{T}$ is the temperature change required to induce a $2 \pi$ phase difference between the two polarizations of the fundamental mode.

The value of the parameter $T_{T}$ multiplied by the length of the fiber $L$ is a wavelengthdependent constant:

$$
L \cdot T_{T}=\operatorname{const}(\lambda)
$$

This dependence previously established for strain measurements using HB bow-tie fibers, enables a range of possible approaches to realizing polarimetric temperature and strain 
sensors by utilizing a variety of HB-PM and PM-PCF fibers that are specifically tailored to particular applications.

The two types of polarization maintaining (PM) fibers used in this study are Panda fiber and photonic crystal fiber (PM-PCF). In the case of the Panda fiber both temperature and strain lead to a phase difference between orthogonal polarizations of the fundamental mode $\mathrm{LP}_{01}$, while in the case of PM-PCF the phase difference arises only from strain as PM-PCF fibers are proven to have a very low temperature sensitivity $[13,9]$ in free space, due to the pure silica structure of PCF.

\section{Fabrication of composite samples with embedded optical fiber sensors}

The fibre glass reinforced composite materials for this study are fabricated by the hand lay-up method, with woven glass fiber fabrics as reinforcement bound together by resin. Samples were prepared with Synolite 1066-P-1, a pre-accelerated, thixotropic, unsaturated polyester resin manufactured by DSM Netherlands having a viscosity of $400 \mathrm{mPa} . \mathrm{s}$, and with a specific gravity of 1.29 at $25{ }^{0} \mathrm{C}$. Plain woven Fiberglass fabrics from JPS Composite Materials Corporation with a density of $280 \mathrm{~g} / \mathrm{m}^{2}$ were used. The dimension of each layer of the fabrics was $215 \mathrm{~mm} \times 120 \mathrm{~mm}$. The fabrication process is as follows: the mould surface is coated with a release agent of an anti-adhesive agent poly vinyl chloride, to prevent sticking the moulded part to the mould surface. The catalyst used is Luperox K1 manufactured by Arkema- France, which is added at a 100:1 resin: catalyst-mix ratio for the hardening process. The first glass fibre reinforcing fabric is placed on the mould and using a brush the fibre fabrics is impregnated with the resin. Then the second layer of the fibre reinforcing fabric is put in place after applying the resin and the process is repeated with subsequent stacked layers until the sample has eight layers of liquid resin and woven reinforcing glass fibre fabrics. The orientation of the fibre glass reinforcement was chosen to provide a $0-0$ ply unidirectional configuration. The fabrication process is shown in Figure 1.

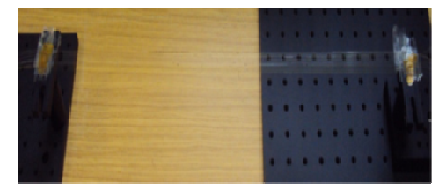

(a)

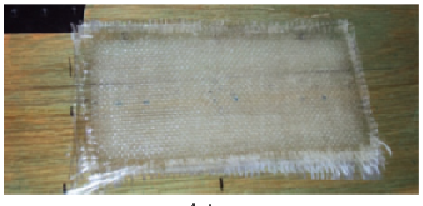

(r)

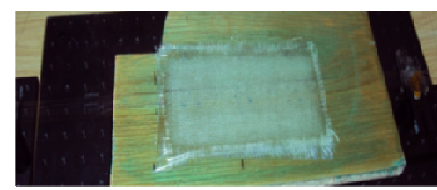

(b)

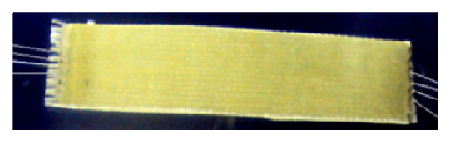

(d)

Figure 1. (a) Pre-strained optical fiber sensors (b) Fiber sensors at the layer $1 \&$ layer 5 of the composite material (c) Composite material with embedded sensors just after fabrication (d)

Cured composite material sample trimmed to the desired shape

The fifth and the first layers are chosen as the locations for the optical fibers placed at a distance of $2 \mathrm{~mm}$ apart from each other. As we had previously observed that the fiber coating may influence the operation of the sensor, the sensing optical fibers are embedded with and without fiber coating for comparison purposes. During the process of embedding and composite curing, a pre-strain is applied to the optical fibers positioned between the composite layers to make sure the fibers remain straight. The composite material sample is cured at $25^{\circ} \mathrm{C}$ for about 20 hours. The composite material samples are uniformly cured and then post-cured [14] at a temperature of $60^{\circ} \mathrm{C}$ for about 8 hours to eliminate the presence of any excess moisture or solvents. The sample is then removed from the mould surface. After curing, the samples are trimmed so they all have common dimensions of $215 \mathrm{~mm}$ x $40 \mathrm{~mm}$ and are $1.8 \mathrm{~mm}$ thick. 
The polarimetric fiber sensors are realized based on two types of highly birefringent fibers: polarization maintaining Panda fiber (PM-1500-HP) and polarization maintaining photonic crystal fiber (PM-1500-01), having beat lengths of $5.0 \mathrm{~mm}$ and $<4 \mathrm{~mm}$ respectively at an operating wavelength of $1500 \mathrm{~nm}$. Both types of fibers have identical acrylate coatings with geometrical parameters as shown in Figure 2(a) and Figure 2(b) respectively.

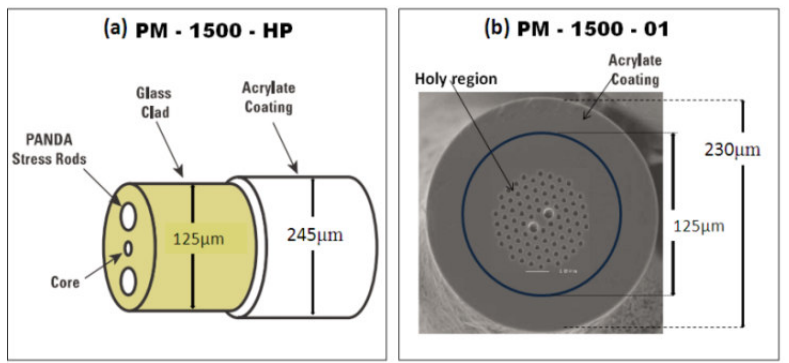

Figure 2. Cross sectional view of highly-birefringent fibers (a) Panda fiber (b) PM-PCF

The embedded positions for the fiber optic sensors within the composite sample layers are shown in cross section in Figure 3. The PM-PCF polarimetric sensor fiber without a coating is positioned at $17 \mathrm{~mm}$ from the left side of the sample. PM-PCF with a coating is positioned 19 $\mathrm{mm}$ from the left side of the sample. The Panda polarimetric sensor fiber without a coating is positioned $21 \mathrm{~mm}$ from the left side. Finally the Panda fiber with a coating is positioned 23 $\mathrm{mm}$ from the left side. Two such sets of identical sensor fiber sets are placed in layer 1 and in layer 5 in order to study the influence of placing the optical sensors in different layers of the composite sample on the sensors performance. Layers 1 and 5 were chosen because they represent the layers closest to the sample surface and the sample centre respectively.

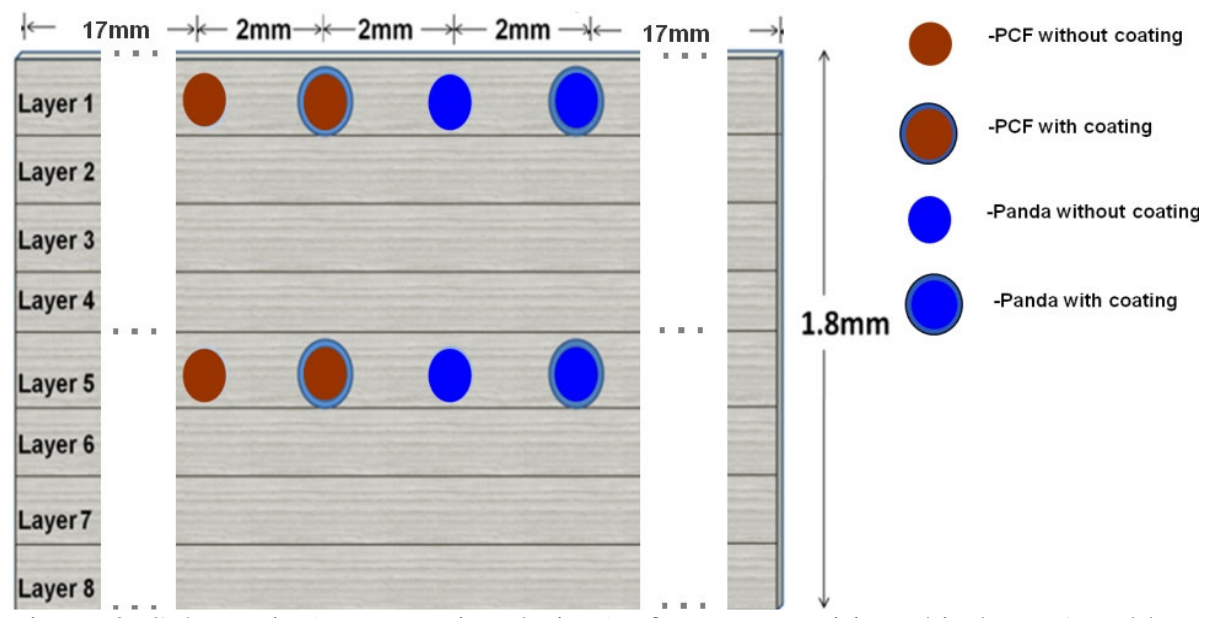

Figure 3. Schematic (cross sectional view) of sensors positioned in layer 1 and layer 5 of the 8-layer composite material sample.

\section{Experimental arrangement}

To extract the phase difference between the two orthogonal polarizations, the experimental setup shown in figure 4 is used. A tunable laser source with a wavelength of $1550 \mathrm{~nm}$ is used as an input source. The temperature sensitivity of the polarimetric sensors is measured by using a polarimeter and polarization control system, TXP 5004 from Thorlabs. The output of the laser source is fed to the polarization controller, so that polarized light is launched into the inputs of the optical fiber sensors. Polarization variations at the outputs are observed by using the polarimeter. It is well known that fiber splicing can induce changes in the polarization state and therefore the response of a polarimetric sensor. Therefore the PM fibers are 
connected without the use of splices, with free ends connected to the polarization control system by means of polarization maintaining connector ferrules.

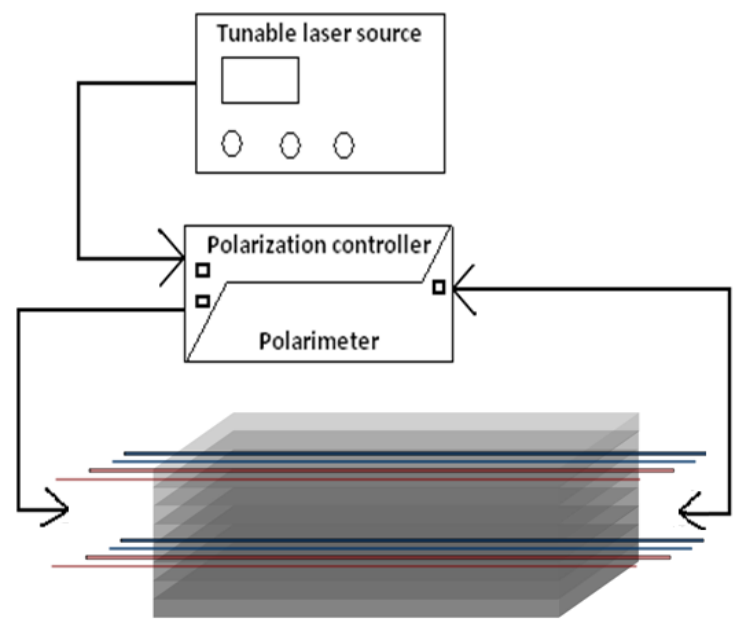

Figure 4. Schematic of the experimental arrangement for composite material sample with embedded polarimetric sensors

To study the influence of the thermal expansion of the composite material on the embedded polarimetric fiber sensors, the sample is subjected to temperature variations over a range from 0 to $65{ }^{\circ} \mathrm{C}$, by using a Peltier cooler (Model No. PE-127-20-15-U from Radionics. Ltd) and the experimental set up as shown in the figure 5. To increase the surface area of the Peltier cooler to match that of the composite sample, a thermally conductive aluminium plate with dimensions of $210 \mathrm{~mm} \times 100 \mathrm{~mm} \times 1.5 \mathrm{~mm}$ is placed on top of the Peltier cooler, which has a surface area of $62 \mathrm{~mm} \times 62 \mathrm{~mm}$, attached with a thermally conducting paste. A time interval of 20 minutes is used between each $5{ }^{\circ} \mathrm{C}$ step temperature variation in order to ensure a uniform temperature distribution in the composite sample. The Peltier cooler temperature is controlled using Thorlabs ITC510 temperature controller with the thermistor feedback sensor attached to the surface of the aluminium plate. The temperature controller offers stability over 0 to $70{ }^{\circ} \mathrm{C}$ operation range, with an accuracy of $0.1^{\circ} \mathrm{C}$. The sample is placed on the aluminum plate so that layer 1 is in contact with the plate.

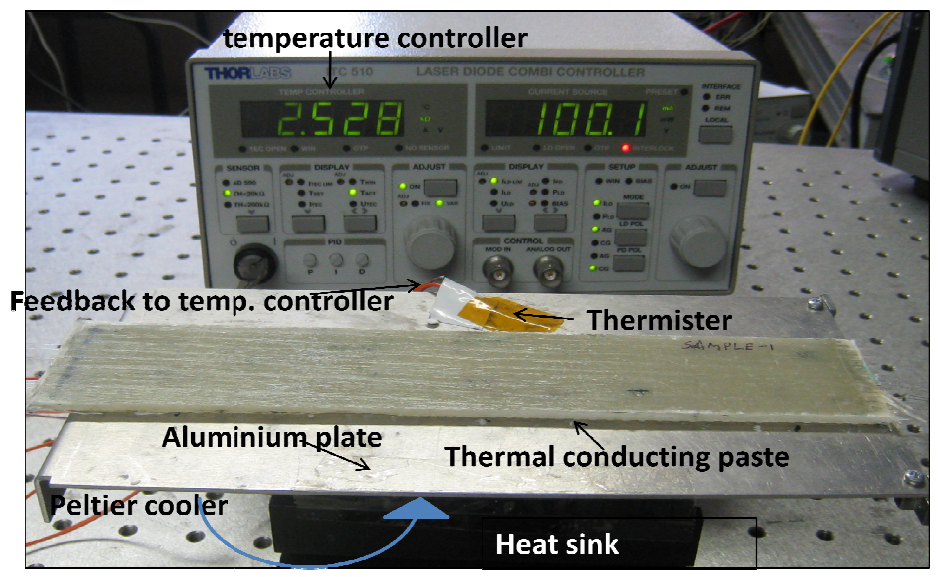

Figure 5. Experimental setup to apply temperature changes to the composite material sample

The strain or temperature induced polarization variation is measured using a polarization controller and polarimeter. The State of Polarization (SOP) [15] at the input to the polarimetric sensors is adjusted by changing the azimuth and ellipticity at the polarization controller. Initially these values are set so as to achieve the maximum optical power at the 
polarimeter input. The Poincare sphere is used as a way to graphically represent the polarization transformations. The Poincare sphere is a graphical tool in real, three dimensional space in which the polarization can be uniquely represented by a point on or within a unit sphere centered in a rectangular $(\mathrm{x}, \mathrm{y}, \mathrm{z})$ coordinate system. The coordinates of the point are the three normalized Stokes parameters describing the state of polarization. Because a state of polarization is represented by a point, a continuous change in the polarization state can be represented as a continuous path on the surface of the Poincare sphere. Thus the strain and temperature response of the polarimetric sensor can be determined by the evaluation of the Poincare sphere.

The polarized light from the source is launched with its polarization at an angle of $45^{\circ}$, to the optical axis of the PM- fiber to ensure equal distribution of optical intensity between the two orthogonal polarizations. The polarimeter provides real time information about the SOP of the polarimetric sensor output, presented graphically as a circular trace on a Poincare sphere with phase change and also numerical values for measurands such as azimuth, ellipticity, Stokes parameters and the intensity of the optical power. The temperature is varied in equal steps, and the variations of the Poincare sphere trace and Stokes parameters are recorded for each step change. The polarization induced phase shift for an applied temperature change is calculated from the Poincare sphere. By extrapolating from the recorded results, the applied temperature needed to obtain one complete circular path on the Poincare sphere (exactly $2 \pi$ radians) is determined and thus the temperature sensitivity can be estimated.

\section{Results and discussion}

\subsection{Temperature response of the embedded PM-PCF sensors}

Temperature responses of both coated and coating-stripped PM-PCF polarimetric sensors embedded in the composite material sample are presented in this section. For comparison the temperature responses of the coating-stripped PM-PCF in free space were found before embedding when subjected to a temperature variation from 0 to $65{ }^{\circ} \mathrm{C}$ and as expected the sensor is found to be effectively temperature insensitive as shown in Figure 6 (a).

However the coating-stripped PM-PCF embedded in the layer 1 of the composite material shows a phase shift of $3.6028 \mathrm{rad}$, for a temperature variation of $65{ }^{\circ} \mathrm{C}$ as shown in Figure 6(b), resulting in a sensitivity of $0.2578 \mathrm{rad} / \mathrm{m} \times{ }^{0} \mathrm{C}$, and for layer 5 a sensitivity of 0.2247 $\mathrm{rad} / \mathrm{m}_{\mathrm{x}}{ }^{0} \mathrm{C}$. Since coating-stripped PM-PCF proved to be insensitive to temperature in free space, it is concluded that the sensitivity when embedded in a composite must be due to strain/stress induced by temperature variations within the composite material or thermal expansion of the composite material.

(a)

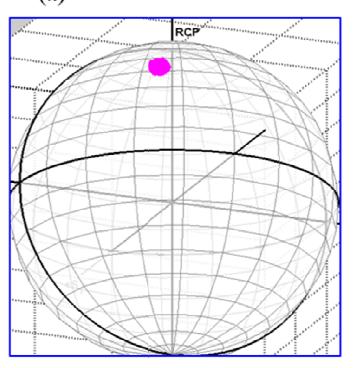

(b)

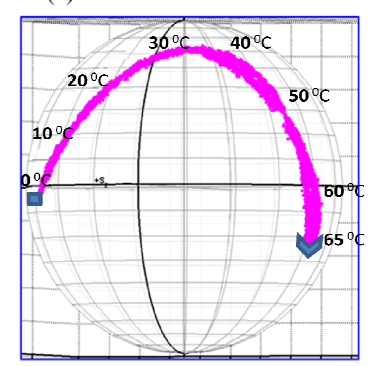

Figure 6. Observed phase shift in coating-stripped PM-PCF fibers for a temperature variation from 0 to $65^{\circ} \mathrm{C}$ (a) in free space (b) after embedding

The process followed above for the coating stripped fibers above was then repeated for the coated PM-PCF fibers. The temperature response of the coated PM-PCF in free space subjected to a temperature variation of $0-65{ }^{\circ} \mathrm{C}$ was firstly determined and is shown in Figure 7 (a). In the coated PM-PCF, a phase shift of $0.1548 \mathrm{rad}$ is observed for a temperature variation of $65^{\circ} \mathrm{C}$, yielding an estimated sensitivity of $0.01108 \mathrm{rad} / \mathrm{m}_{\mathrm{x}}{ }^{0} \mathrm{C}$. While this value for free space is low, it is non-zero, unlike the coating stripped PM-PCF. When the coated 
PM-PCF is embedded in layer 1 of the composite material sample (and therefore is in direct contact with the heat source) and is subjected to a temperature variation of $65{ }^{\circ} \mathrm{C}$ it shows a phase shift of $0.1518 \mathrm{rad}$ (Figure 7 (b)), equivalent to a sensitivity of $0.01086 \mathrm{rad} / \mathrm{m}^{0} \mathrm{C}$. For a coated PM-PCF embedded in the layer 5 the temperature sensitivity also yields a comparable low value of $0.0185 \mathrm{rad} / \mathrm{m}^{0}{ }^{0} \mathrm{C}$. Comparing the free space and embedded sensitivities it is clear that for a coated PM-PCF the sensitivity is not unduly affected by embedding, a very different conclusion in comparison to that for the coating-stripped case, where sensitivity increases dramatically after embedding.

(a)

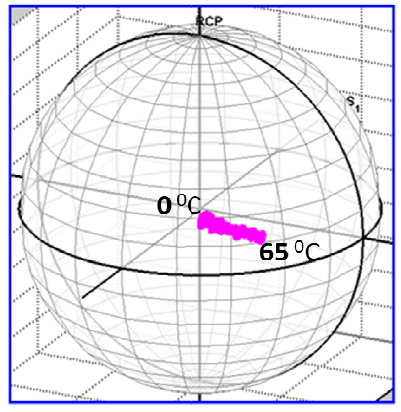

(b)

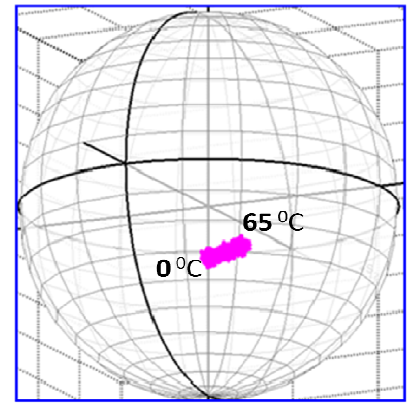

Figure 7. Observed phase shift in coated PMPCF fibers for a temperature variation from 0 $65^{\circ} \mathrm{C}$ (a) free space (b) after embedding

It should be noted that by comparing the performance of coating-stripped and coated PM-PCF in free space from Figure 6(a) and Figure 7(a), it is observed that there is a minor dependence on temperature for the buffer coated PM-PCF, which we believe arises due to the temperature sensitive nature of the acrylate coating of the PM-PCF fibers.

We believe that the difference in the fiber sensor temperature sensitivity between coating stripped PM-PCF and a coated PM-PCF when embedded in the composite is due to the higher tolerance of the coated PM-PCF to the dominant form of thermal expansion in a composite material, that is transverse expansion. During cooling and heating cycles the SOP followed the same trace indicating the absence of a hysteresis phenomenon, confirming also that the 0 $65^{\circ} \mathrm{C}$ experimental temperature range lies in the linear thermal expansion region of the composite material, which is reasonable given that the glass transition temperature of the particular cured resin is above $150{ }^{\circ} \mathrm{C}$. It is identified in [16] that composite materials have both longitudinal and transverse expansions, but that the coefficient of transverse thermal expansion is many times higher than that for longitudinal expansion.

The thermal expansion induced stress and the strain, causes a change in the birefringence of the PM-PCF and as a result a phase shift between the guiding modes can occur in the case of a buffer stripped PM-PCF embedded in the composite material. However for the coated PMPCF sensor, the impact of the thermal expansion of the composite material has a much lower effect as seen in Figure 7 and as a consequence the temperature sensitivity of the sensor by comparison to the free space sensitivity does not vary significantly after embedding in the composite material. This immunity to thermal expansion in a buffer coated PM-PCF is due to two reasons: (i) the impact of transverse thermal expansion is eliminated due to strain tolerance provided by the coating material, thus no stress induced birefringence change arises (ii) the coefficient of longitudinal thermal expansion of the glass reinforced general purpose resin based composite is very low [17].

\subsection{Temperature response of the embedded panda fiber sensors}

The temperature responses of both coated and coating-stripped panda fiber polarimetric sensors embedded in the composite materials sample are presented in this section. A panda fiber based polarimetric sensor is sensitive to both temperature and strain. To analyze 
temperature sensitivity the panda fiber samples are subjected to temperature variations without applying any strain.

For the buffer coating stripped case, the temperature responses of the panda fiber in free space and embedded in the layer 1 of the composite material were measured and show a phase shift of $3.1605 \mathrm{rad}$ and $6.175 \mathrm{rad}$ respectively, when subjected to a temperature variation $0-7^{\circ} \mathrm{C}$, as shown in Figure 8(a) and Figure 8(b). Note that since panda type fibers are more sensitive compared to PM-PCF, in order achieve a phase variation within one complete circuit of a Poincare sphere the smaller temperature range of $0-7{ }^{0} \mathrm{C}$ is used to enable a more direct comparison with the PM-PCF case. A significant change in temperature sensitivity is observed before and after embedding of coating stripped panda fibers into composite materials similar to that of the PM-PCF. The magnitude of temperature sensitivity increased from $2.1 \mathrm{rad} / \mathrm{m}^{0}{ }^{0} \mathrm{C}$ to $4.1 \mathrm{rad} / \mathrm{m}^{0}{ }^{0} \mathrm{C}$. The coating stripped panda fiber that is embedded in the composite layer 5 yielded a temperature sensitivity value of $3.56 \mathrm{rad} / \mathrm{m} \times{ }^{0} \mathrm{C}$. This difference in the magnitude of the temperature sensitivity before and after embedding confirms the dependence of the coating-stripped panda fiber temperature response on composite material thermal expansion.

(a)

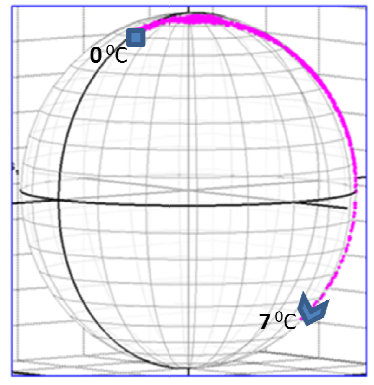

(b)

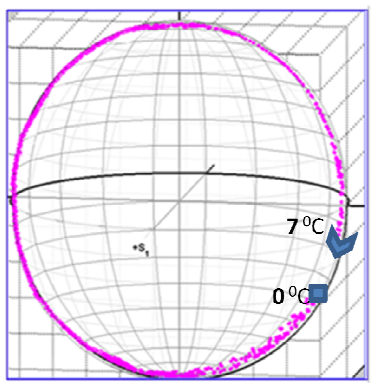

Figure 8. Observed phase shift in coating-stripped Panda fibers for a temperature variation from $0-7^{0} \mathrm{C}$ (a) free space (b) after embedding

For the buffer coated case, investigations were also undertaken for the panda fiber in free space and embedded in the composite material sample. For layer 1 and a temperature variation of $7{ }^{\circ} \mathrm{C}$, there is a phase shift of $3.311 \mathrm{rad}$ and $3.913 \mathrm{rad}$ respectively as shown in Figure 9(a) and Figure 9(b).The corresponding temperature sensitivities are $2.2 \mathrm{rad} / \mathrm{m} \times{ }^{0} \mathrm{C}$ and $2.6 \mathrm{rad} / \mathrm{m} \times{ }^{0} \mathrm{C}$. The coated panda embedded in the composite layer 5 yields a value of $2.34 \mathrm{rad} / \mathrm{m} \times{ }^{0} \mathrm{C}$. Thus the magnitude of temperature sensitivities for buffer coated panda fiber sensors are comparable in free space and after embedding, from which we can conclude that there is no significant influence for coated panda fiber due to the thermal expansion of the composite material.

(a)

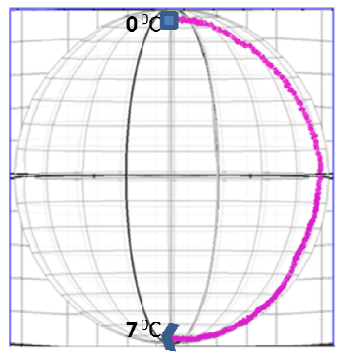

(b)

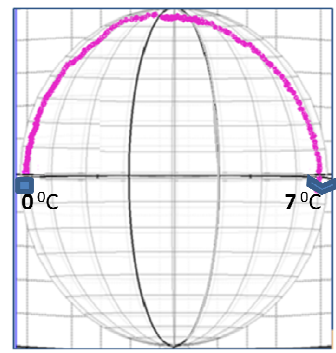

Figure 9. Observed phase shift in coated Panda fibers for a temperature variation from $0-7^{0} \mathrm{C}$ (a) free space (b) after embedding

Comparing the performance of coating-stripped and coated panda fibers in free space as shown in Figure 8. (a) and Figure 9. (a) respectively, the reason for the slight increase in temperature sensitivity from 2.1 to $2.2 \mathrm{rad} / \mathrm{m}^{0}{ }^{0} \mathrm{C}$ for coated panda fiber in free space is due to 
the acrylate coating which undergoes a small thermal expansion which induces low levels of stress on the fiber.

A comparison of the temperature responses of embedded panda fiber without a coating Figure 8. (b) and with a coating Figure 9(b), shows significant difference in sensitivities from 4.1 $\mathrm{rad} / \mathrm{m \times}{ }^{0} \mathrm{C}$ to $2.6 \mathrm{rad} / \mathrm{m} \times{ }^{0} \mathrm{C}$ respectively. The effective temperature sensitivity when embedded of a buffer stripped Panda fiber is significantly higher compared to a coated panda fiber due to the influence of transverse thermal expansion, as explained in section 5.3.

\subsection{Discussion}

Figure 10(a) summarises and compares the temperature sensitivities of PM-PCF sensors in various configurations. It is clear that the presence of a coating preserves the temperature independence of a PM-PCF sensor when it is embedded in a composite. In a similar fashion, Figure 10(b) summarises and compares the temperature sensitivities of panda fiber sensors in various configurations. The inherent temperature sensitivity of panda fiber sensors is higher, but once again the presence of a coating, mitigates the effect of thermal induced stress in the composite. A conclusion is that while a coating stripped PM-PCF sensor can be used as a temperature independent strain sensor in free space, it cannot be used for strain measurements in composite materials. The acrylate coated panda sensors can be used for embedding in composites provided a temperature correction is used to increase the accuracy of strain measurements. However for embedding in composite materials, coated PM-PCF sensors are most effective in delivering temperature insensitive strain measurement.

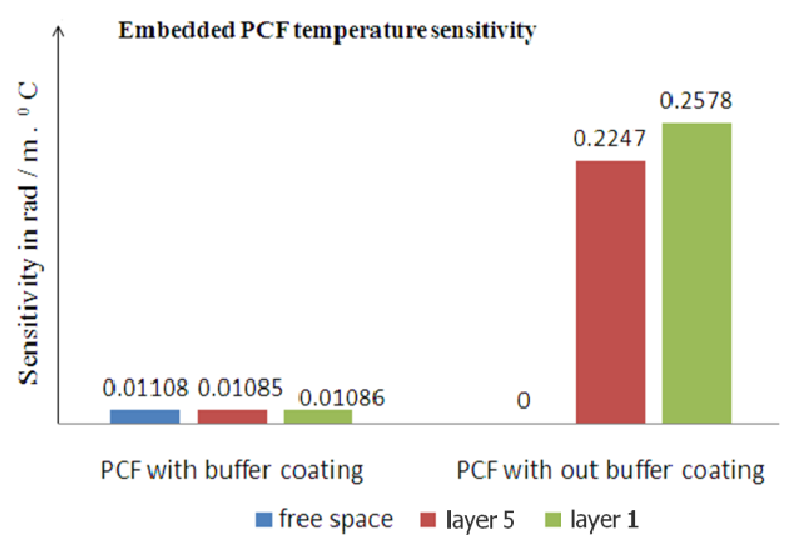

(a)

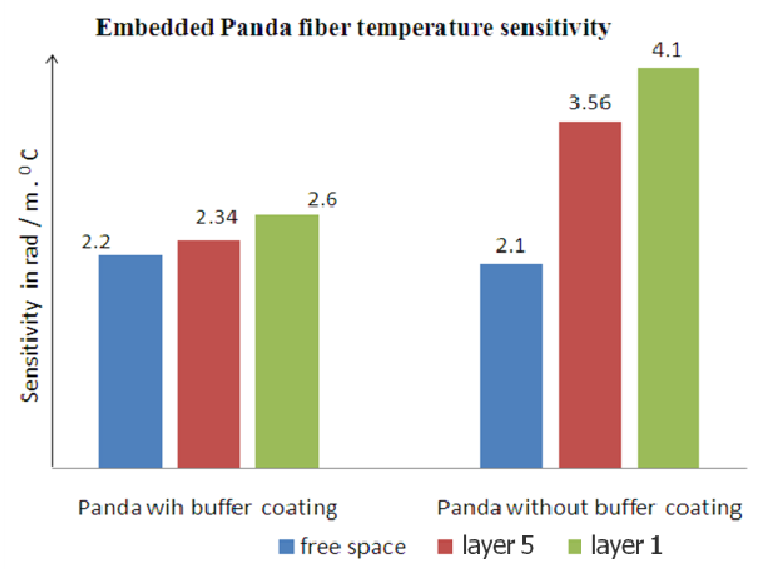

(b)

Figure 10. Comparison of the temperature sensitivity of (a) PM-PCF polarimetric sensors

(b) Panda polarimetric sensors 
One final insight from Figure 10 is that optical fiber sensors in the outer layer of a composite sample show a higher thermal stress sensitivity due to the fact that the temperature gradually decreases in the direction from the outer layer that is contact with the heat source toward the layer 5 , with a consequent reduction in thermally induced stress.

\section{Conclusion}

The vital issues of the influence of composite material thermal expansion on embedded polarimetric sensors for measurements of strain and temperature are studied in this paper. The glass-fiber reinforced general purpose resin based composite samples are fabricated and characterized, with polarimetric sensors embedded in two distinct layers of the multi-layer composite structure. The two types of polarimetric sensors studied are based on polarization maintaining photonic crystal fiber and panda fiber. The temperature sensitivities of coated and coating stripped PM-PCF and panda fiber polarimetric sensors are calculated in free space and compared to those of embedded in the composite material. From the results it is concluded that a polarimetric sensor with an acrylate coated fiber embedded in the composite material shows the same response as the one in free space, while a coating-stripped fiber polarimetric sensor shows a significant increase in temperature sensitivity when embedded in the composite material, which is due to the stress induced change in birefringence created by thermally induced strain. In the case of coated fiber, the effect is being eliminated by the coating. For panda fibers the magnitude of dependency on thermal expansion of the composite material is many times higher than for PM-PCF, because of the cross-sensitivity between temperature and thermal induced strain. From the analysis it is concluded that thermal expansion induced drifts are the main source or errors during temperature and stain measurements using embedded polarimetric sensors. To improve the sensor system accuracy in real-world applications, it is concluded that buffer coated polarimetric fiber sensors are a better choice than buffer stripped fiber sensors.

\section{Acknowledgements}

This research is supported by Enterprise Ireland under the international research grant MATERA ERA-NET.

\section{References}

[1] Chung D D L 2010 Composite materials: science and applications Springer Publications $2^{\text {nd }}$ Edition

[2] Grassi M, Zhang X, and Meo M 2002 Prediction of stiffness and stresses in Z-fiber reinforced composite laminates Composite Part A: applied science and manufacturing vol 33 pp 16531664.

[3] Kim J K, Wo D Z, Zhou L M, Huang H T, Lau K T, and Wang M 2007 Advances in Composite Materials and Structures Key Engineering Materials vol 334-335( 2007).

[4] Garg D P, Zikry M A, Anderson G L, Gros X E 2001 Current and potential future research activities in adaptive structures: an ARO perspective Smart Mater. Struct. vol 10 pp 610-623.

[5] Dewynter-Marty V, Ferdinand P, Bocherens E, Carbone R, Beranger H, Bourasseau S, Dupont $\mathrm{M}$ and Balageas D 1998 Embedded fiber Bragg grating sensors for industrial composite cure monitoring Journal of Intelligent Materials Systems and Structures vol 9 pp 785-787

[6] MurukeshanV M Chan P Y, Seng O L, and Asundi A 1999 On-line health monitoring of smart composite structures using fiber polarimetric sensor Smart Mater. Struct. vol 8 pp 544-548.

[7] Yoshino T, Hashimoto T, Nara M, and Kurosawa K 1992 Common path heterodyne optical fiber sensors J. Lightwave Technology vol. 10 pp. 503-513, Apr. 1992

[8] Ashish M, Vengsarkar, Craig Michie W, Ljilja Jankovic, Brian Culshaw and Richard O C 1994 Fiber-optic Dual-Technique Sensor for Simultaneous Measurement of Strain and Temperature J. Lightwave Technology vol. 12 No: 1,Jan. 1994.

[9] Rajan G, Mileńko K, Lesiak P, Semenova Y, Boczkowska A, Ramakrishnan M, Jędrzejewski K, Domański A, Woliński T, and Farrell G 2010 A hybrid fiber optic sensing system for 
simultaneous strain and temperature measurement and its applications Photonics Letters Of Poland, vol 2 (1) pp 46-48.

[10] Villatoro J, FinazziV, Minkovich V P, Pruneri V, and Badenes G 2009 Highly sensitive sensors based on photonic crystal fiber modal interferometers Journal of Sensors vol 2009 Article ID 747803.

[11] Domanski A Wet al 2009 Comparison of Bragg and Polarimetric Optical Fiber Sensors for Stress Monitoring in Composite Materials Acta Physica Polonica vol 116 p 294.

[12] Wolinski T R, Lesiak P, Domanski A W, 2008 Polarimetric optical fiber sensors of a new generation for industrial applications Bulletin Of The Polish Academy Of Sciences Technical Sciences vol 56 No. 2.

[13] Dong X, and Tam H Y2007 Temperature-insensitive strain sensor with polarization maintaining photonic crystal fiber based Sagnac interferometer Applied Physics Letters vol 90 pp 151113 - 151113-3

[14] Delaye N, Marais S 1998 Characterization of unsaturated polyester resin cured with styrene J Appl Polym Sci 1998;67:695-703.

[15] Collett E Feild guide to polarization 2005 SPIE presss SPIE field guides vol FG05.

[16] Reid S R, and Zhou G 2000 Impact behaviour of fibre-reinforced composite materials and structures CRC press October 31(2000).

[17] Stolarski T, Nakasone Y, Yoshimoto2007 A numerical study on the coefficients of thermal expansion of fiber reinforced composite materials Composite Structures vol 78 pp 1-10. 